\title{
Numerical Simulation about the Effect of Stator Pitch and Stator Thickness on Contact Pressure of Progressive Cavity Pump
}

\author{
Weiyue $X u^{1, a^{*}}$, Zungang Yuan ${ }^{2, b}$ and Guiling Deng ${ }^{3, c}$ \\ ${ }^{1}$ Central South University, Changsha, China \\ 2 Shen Zhen Xetar Technology Co.,Ltd., Shenzhen, China \\ ${ }^{3}$ Central South University, Changsha, China \\ a18573156530@163.com, bxyd@xetar.com.cn, c gldeng@csu.edu.cn
}

\begin{abstract}
Keywords: finite element analysis, conventional PCP, even-thickness PCP, stator pitch, stator thickness, contact pressure.

Abstract: Progressive cavity metering pump(PCP) which is especially good for the transmission of high viscosity liquids containing solid grains has been wildly used in electronic packaging industry in recent years. Due to the existence of pipeline pressure, adequate hydraulic sealing pressure is necessitated to prevent back-flow especially in high accuracy liquid transmission situations. There is a positive correlativity between hydraulic sealing pressure and contact pressure of PCP, so three-dimensional finite element models of PCP with high quality hexahedral mesh divided by ANSYS-ICEM is established to study the effect of stator pitch and stator thickness on contact pressure of PCP.
\end{abstract}

\section{Introduction}

Since the PCP was invented by Moineau in 1930, PCPs have been more and more used for pumping high viscosity fluids or slurries, mainly in food and cosmetic industry, but it is the growth of the application of these pumps for oil artificial lifts in low to medium depth oil wells since the 1970s (in several cases substituting for traditional reciprocating pumps) that leads to the development of more detailed experimental studies within these devices [1].

So far, we still haven't understood the performance of PCPs completely. Noonan mentioned our lack of adequate cognition of PCP's applications and operating range and proposed that "the industry is striving to understand PCP performance in a variety of environments and operational limits". She also added that "while the industry is much further ahead on the PCP learning curve as they were 10 years ago, there is still much that needs to be understood, especially pump pressure capability, and PCP system reliability" [2]. The pump pressure capability is one key performance parameters of PCPs, for the excellent pump pressure capability can effectively prevent internal slip which will lead to bad measuring accuracy.

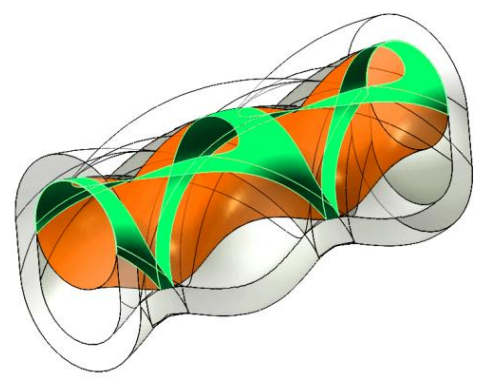

(a)

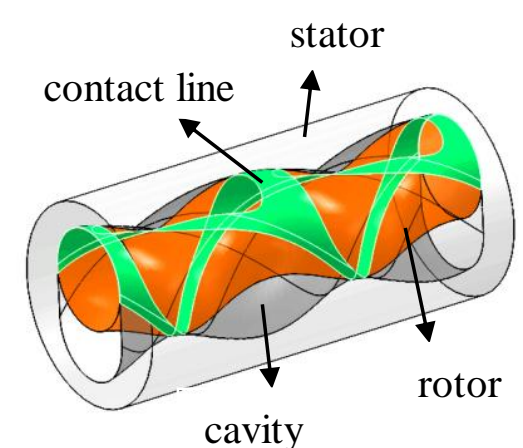

(b)

Fig. 1. Schematic images of even-thickness PCP (a) and conventional PCP (b).

Research works about the contact pressure between stator and rotor have been carried out all the time. but the contact stress line will be hardly continuous in the post-processing if the mesh quantity is not enough, however, large quantities of mesh will bring about tremendous amount of calculating time. In order to overcome above shortcomings, 3D finite element models of conventional and 
even-thickness PCPs with full hexahedral mesh were established to study the effect of stator pitch and thickness on contact pressure in this paper.

\section{PCP Operation Principle}

A progressive cavity pump is a rotary positive displacement pump which consists of a spiral shaped rotor that moves eccentrically inside the stator. The stator has the shape of an internal gear with one tooth more than the rotor, and it is made of rubber often, the rotor coated with chrome is made of steel usually [3]. When the rotor is positioned in the stator, the contacting locations between these two components create a series of cavities(as is shown in Fig.1). The eccentric motion of the rotor displaces the liquid in the cavities spiral around the outside of the rotor from low to high pressure regions. Thus hydraulic seals are required between rotor and stator to avoid back-flow (from high to low pressure regions), which will lower the pump efficiency.

\section{Contact Pressure Simulation}

\subsection{Finite Element Model}

The article took the PCP GLB120-27 with stator pitch of $80 \mathrm{~mm}$, eccentricity of $5 \mathrm{~mm}$, rotor diameter of $38.6 \mathrm{~mm}$ and interference of $0.3 \mathrm{~mm}$ as the research object, and focused on the effect of stator pitch and thickness on contact pressure. So six major stator diameters and five stator pitches in both even-thickness and conventional PCP finite element models were considered in this paper, being $66,70,73.5,77,80,83 \mathrm{~mm}$ and $76,78,80,82,84 \mathrm{~mm}$ respectively.

Rubber is usually used to made the stator of PCP, as a kind of nonlinear elastic material, there exists two kinds of constitutive model about it, in the first kind, rubber material is regarded as an incompressible material, strain energy density is supposed to be a polynomial function of principal strain invariants, the model will be called Mooney-Rivlin model if the first degree of the function is employed only. In the second kind, strain energy density is supposed to be an independent function of three main elongation, such as Ogden, Peng and Peng-Landel material model [4].

The physical nonlinearity of rubber is usually described by Mooney-Rivlin model in simulation. The strain energy density function can be expressed as:

$$
W\left(I_{1}, I_{2}\right)=\sum_{i, j=0}^{n} C_{i j}\left(I_{1}-3\right)^{i}\left(I_{2}-3\right)^{j}
$$

Where $\mathrm{W}$ is strain energy density; $\mathrm{C}_{\mathrm{ij}}$ is Rivlin coefficient which can be got from kinds of methods, such as uniaxial tensile test and hardness test; $I_{1}, I_{2}$ are the first and second Green strain invariants respectively.

Taking the advantages of structural cycle-symmetry of PCP and tremendous disparity in elastic

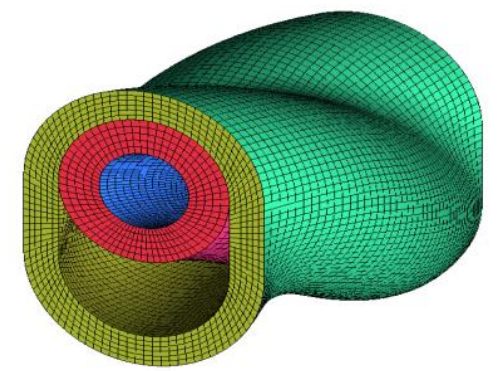

Fig. 2. Finite element model of even-thickness PCP with half stator pitch

modulus between steel rotor and rubber stator, half pitch length solid model with hollow rotor was modeled in SOLIDWORKS for the sake of reducing the number of mesh and calculation time. Then 
the solid model was imported to mesh generation software ANSYS-ICEM, mesh assembly method was adopted in the process of finite element model building, namely that mesh dividing of stator and rotor was accomplished in advance, then imported the mesh files of them into assembly model. O-block of ANSYS-ICEM is especially fit for dividing stator and rotor, (Fig.2 shows the mesh model of assembly of stator and rotor) finally exported the mesh model of assembly to ANSYS for other settings.

In ANSYS, Solid185 and Solid 45 were selected to be the element type of stator and rotor respectively, define $\mathrm{C} 10=-7.513 \times 105, \mathrm{C} 01=-2.375 \times 106$ in Mooney-Rivlin model of stator [5], set the rotor as linear elastic material whose mechanical parameters is the same as steel. According to contact pair setting regulations when contact pair is composed of soft face and hard face, the soft face should be selected as contact face. So the inner face of stator was set as contact face, and the outer face of rotor was chosen to be target face.

\subsection{Initial Condition and Boundary Condition}

The stator rubber is bonded to an outer steel tube, so fixed constraints should be loaded on the outer surface of stator. Fixed constraints was also loaded on both end face of rotor, and symmetry constraints was loaded on both end face of stator. Some scholars made the rotor diameter larger than the size of stator's arc region in solid modeling, attempting to use geometric interference to simulate the interference in computational simulation, but this method is not accurate, for the actual interference between rotor and stator is not the same as geometric interference after meshing.

In order to simulate the interference more accurately, following method can be adopted in ANSYS:

- First, set KEYOPT(9)=4,then ANSYS will include the defined contact surface offset (CNOF) only and take no account of the initial penetration due to geometry in calculation, besides, contact surface offset will be applied step by step during calculation.

- Second, set the real constant ICONT to specify a small initial contact closure to make the nodes of contact face just touch target face.

- Third, set CNOF equal to the interference as you want.

\subsection{Results of Simulation}

For the brevity of this paper, not all the contour curves of contact pressure are included in the article. It is necessary to point out that there exists some difference in the size of inner diameter of hollow rotor used between the thickness research and stator pitch research, this is to say, the inner diameter of rotor in thickness research is $28 \mathrm{~mm}$, and $20 \mathrm{~mm}$ in stator pitch study. This is done because it is found that failure always occurs in mesh partition in models of thickness research when the inner diameter of hollow rotor is $28 \mathrm{~mm}$, but success can be achieved easily when inner diameter is larger

arc contact region

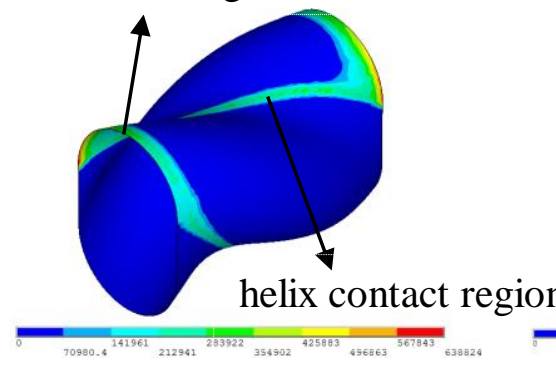

(a)

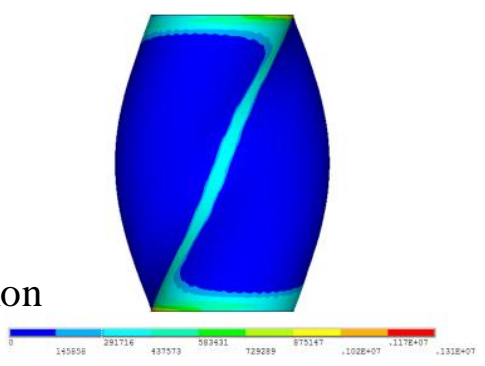

(b)

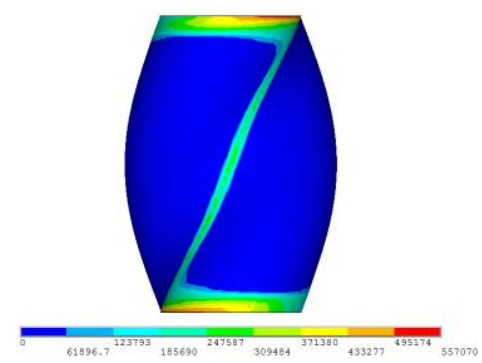

(c)

Fig. 3. Isometric view of contact pressure contour curve of even-thickness PCP (a) when the thickness is $7.75 \mathrm{~mm}$ (major diameter is $73.5 \mathrm{~mm}$ ), Top view of contact pressure contour curve of even-thickness PCP (b) and conventional PCP (c) when the thickness is $7.75 \mathrm{~mm}$ ( their major diameter are $73.5 \mathrm{~mm})$ 
than $20 \mathrm{~mm}$, but this difference will contribute almost no influence to the results of simulation due to the huge disparity in elastic modulus between steel rotor and rubber stator.

Fig.3 (a)shows the isometric view of contact pressure contour curve that belongs to even-thickness PCP with thickness of $7.75 \mathrm{~mm}$ (whose major diameter is $73.5 \mathrm{~mm}$ ). For the convenience of observing distribution of contact pressure, Fig.3(b) and Fig.3 (c) show the top view of the contact pressure contour curve of even-thickness and conventional PCP respectively. It reveals that the contact pressure in helix region of even thickness PCP is higher than that of conventional PCP, besides, the contact pressure distribution in arc region of even-thickness PCP is more uniform than that of conventional PCP.

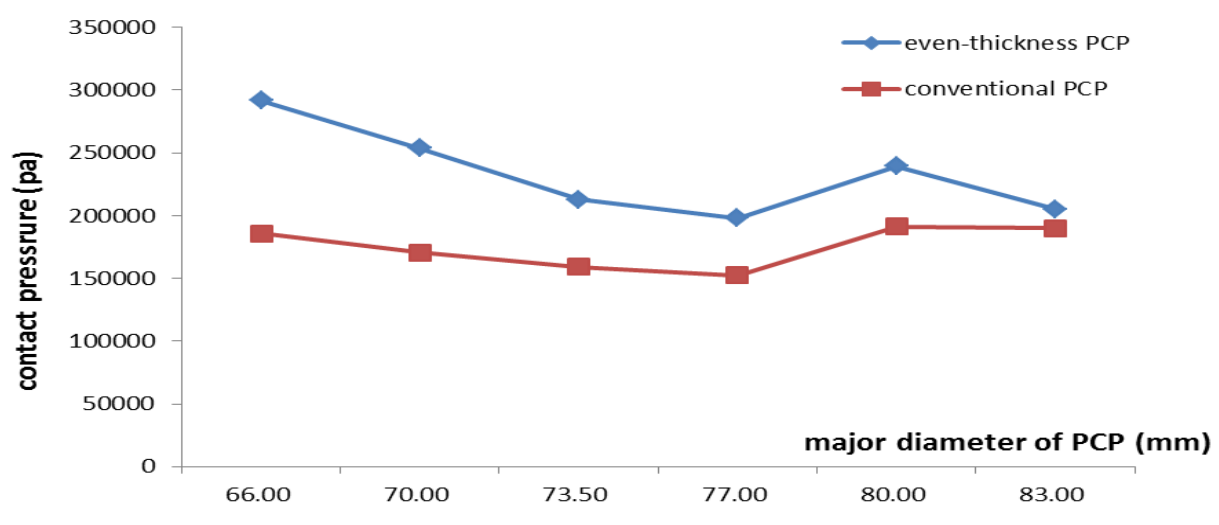

Fig. 4. Contact pressure of PCP with different major diameters

Fig.4 shows the contact pressure varying with the stator thickness( or major diameter) in conventional PCP and even-thickness PCP when the stator pitch is an fixed value of $80 \mathrm{~mm}$. For even-thickness PCP, the contact pressure presents a decreasing trend with the increase of thickness, but for conventional PCP, this trend seems not to be as obvious as the former. In addition to this , contact pressure of even-thickness PCP is larger than that of conventional PCP under the same major diameter. The main reason about this may be that the elastic deformation of even-thickness PCP is more evenly compared with conventional PCP, and the thinner the rubber layer is ,the larger strain would take place, thus bigger contact pressure can be realized under the same interference, which will improve the sealing capacity of PCP.

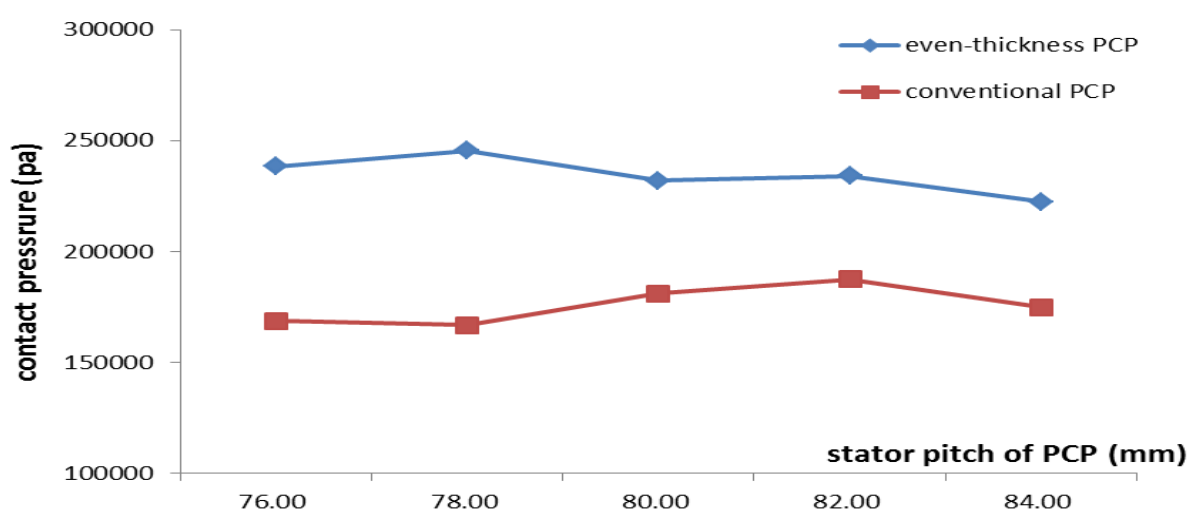

Fig.5. Contact pressure of PCP with different stator pitch

Fig. 5 shows stator pitch has no obvious influence on contact pressure no matter in even-thickness PCP or conventional PCP when stator pitch varies from 66 to $80 \mathrm{~mm}$, and it again reveals that the contact pressure of even-thickness PCP is larger than that of conventional PCP. 


\section{Conclusions}

In this paper, 3d finite element models of even-thickness PCP and conventional PCP with high-quality hexahedral mesh were established to calculate the contact pressure between stator and rotor, correct interference was simulated in these models by setting relevant parameters in ANSYS. Several shortcomings of past models are overcame in this model. The results shows contact pressure in even-thickness PCP is larger than that of conventional PCP under the same major diameter, and trends down with the increase of the thickness of elastomer stator in even-thickness PCP. Besides, the simulation reveals that the contact pressure distribution in even-thickness PCP is more uniform than that of conventional PCP, and small fluctuation of stator pitch will not bring about obvious contact pressure changes in both even-thickness and conventional PCP, but it can't conclude that stator pitch have no influence on contact pressure, for stator pitch changes slowly in this simulation, whether dramatic stator pitch changes will bring regular change of contact pressure is still needed to be simulated.

\section{Acknowledgments}

This work was supported by Shen Zhen Xetar Technology Co.,Ltd., the National Basic Research Program of China (973 program: 2011CB013104) and Fundamental Research Funds for Central Universities of Central South University (2013zzts034), China.

\section{References}

[1] Emilio E. Paladino, João A. Lima, Paulo A.S. Pessoa, Rairam F.C. Almeida, A computational model for the flow within rigid stator progressing cavity pumps, Journal of Petroleum Science and Engineering, vol.78, July 2011, pp.178-192.

[2] Noonan, S. G, The progressing cavity Pump operating envelope: you cannot expand what you don't understand, International Thermal Operations and Heavy Oil Symposium. 2008.

[3] Martin, A. M., Kenyery, F., Tremante, A., Experimental study of two phase pumping in progressive cavity pumps, SPE Annual Technical Conference and Exhibition. Society of Petroleum Engineers, 2002.

[4] Tonglong Chu. Screw pump inner flow field analysis and three-dimensional numerical simulation research, Master degree thesis of Northeast Petroleum University, 2014

[5] Guiying Xu. Research on the influential factors of lifting pressure and parametric optimization of progressing cavity pump, Master degree thesis of Northeast Petroleum University, 2014 